International Journal of Pure and Applied Mathematics

Volume 86 No. 5 2013, 871-881

ISSN: 1311-8080 (printed version); ISSN: 1314-3395 (on-line version)

url: http://www.ijpam.eu

doi: http://dx.doi.org/10.12732/ijpam.v86i5.10

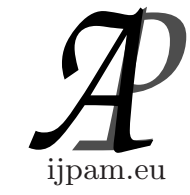

\title{
ON COMMUTATIVE EKFN-RING WITH ASCENDING CHAIN CONDITION ON ANNIHILATORS
}

\author{
Mohameth Alassane Ndiaye ${ }^{1}$, Cheikh Thiécoumba Gueye ${ }^{2} \S$ \\ ${ }^{1,2}$ Laboratoire d'Algèbre, de Cryptographie \\ De Géométrie Algébrique et Applications (LACGAA) \\ Département de Mathématiques et Informatique \\ Faculté des Sciences et Techniques \\ Université Cheikh Anta Diop \\ BP 5005 Dakar, SÉNÉGAL
}

\begin{abstract}
We consider the class $\mathcal{E}$ of endo-noetherian modules, ie the modules $M$ satisfying the ascending chain condition for endomorphic kernels: the chain $\operatorname{Ker} f_{1} \subset \operatorname{Ker} f_{2} \subset \cdots \subset \operatorname{Ker} f_{n} \subset \cdots$ is stationary, where $f_{i} \in \operatorname{End}(M)$. And let $\mathcal{N}$ be the class of noetherian modules. It is well known that every noetherian $R$-module $M$ is endo-noetherian, but the converse is not true, so $\mathcal{N} \subset \mathcal{E}$. The aim of this work, is to characterize commutative rings for which $\mathcal{E}$ and $\mathcal{N}$ are identical.
\end{abstract}

\section{AMS Subject Classification: 13-XX}

Key Words: annihilator, Artinian, EKFN-ring, endo-noetherian, Hopfian, Noetherian, strongly Hopfian

\section{Introduction}

The ascending chain condition is a mathematical property on orders, initially identified by Emmy Noether in 1921 [5] in the context of commutative algebra, and objects satisfying it are named noetherian in her honor. Noether showed that this ascending chain condition is equivalent to the non-existence of an infinite strictly increasing sequence of ideals. These rings named, noetherian rings,

Received: May 20, 2013

(c) 2013 Academic Publications, Ltd.

$\S$ Correspondence author url: www.acadpubl.eu 
have been the subject of extensive study to modules. The descending chain condition on ideals was introduced by Artin. Rings in which any descending chain of ideals is finite are called artinian. We say that a module is noetherian (resp. artinian) if any ascending (resp. descending) chain of submodules is stationary. In fact, the finiteness of the dimension of a vector space $E$ is also characterized by the following properties:

(i) any surjective endomorphism is injective;

(ii) any injective endomorphism is surjective.

Condition (i) (resp. (ii)) has inspired, the concept of hopfian modules (resp. cohopfian modules). An $R$-module is said hopfian if any surjective homomorphism from $M$ to $M$ is injective. This concept began with the introduction of hopfian groups by G. Baumslag in 1963 [7]. Since we focused on other issues relating to hopfian and co-hopfian properties, including:

1. the characterization of rings over which every hopfian (resp. co-hopfian, resp. fitting) module is noetherian (resp. artinian, resp. of finite length);

2. the characterization of rings for which every hopfian (resp. co-hopfian) module is finitely generated.

The question in 1 has been fully resolved, in the commutative case by A. Kaidi and M. Sangharé, who have established in 1988 the following results in [3]: let $R$ be a commutative ring, then the following conditions are equivalent:

(a) $R$ is an $I$-ring; ${ }^{1}$

(b) $R$ is an $S$-ring; ${ }^{2}$

(c) $R$ is an artinian principal ideal ring.

And then in 1992 M. Sangharé has established in [10]: let $R$ be a commutative ring, then the following conditions are equivalent:

(a) $R$ is an $I$-ring;

(b) $R$ is an $S$-ring;

(b) $R$ is an $F$-ring; ${ }^{3}$

\footnotetext{
${ }^{1}$ An $I$-ring is a ring such that every hopfian module is noetherian.

${ }^{2} \mathrm{An} S$-ring is a ring such that every co-hopfian module is artinian.

${ }^{3} \mathrm{An} F$-ring is a ring such that every fitting module is of finite length.
} 
(c) $R$ is an artinian principal ideal ring.

The characterization of the so called FGS-rings, that is those rings for which every hopfian module is finitely generated, was fully resolved in the commutative case, by C.T. Gueye and M. Sangharé in 2004 [4]. They established that: a ring $R$ is an $F G S$-ring if and only if $R$ is an artinian principal ideal ring.

Then the characterization of the so called FGI-rings, that is those rings for which every co-hopfian module is finitely generated, was fully resolved in the commutative case by M. Barry et all in 2005 [9], they established that: a ring $R$ is an FGI-ring if and only if $R$ is an artinian principal ideal ring. Nevertheless, the question remains open in the general case.

In 2007 A. Hmaimou et all introduced in [1], the notions of modules that involve both the conditions of chains and endomorphism, defined as follows: an $R$-module $M$ is said to be strongly hopfian (resp. strongly co-hopfian) if for every endomorphism $f$ of $M$, then Kerf $\subset \operatorname{Ker} f^{2} \subset \cdots($ resp. Imf $\supset$ $\left.\operatorname{Im} f^{2} \supset \cdots\right)$ is stationary. They showed that the class of strongly hopfian (resp. strongly co-hopfian) modules is between the class of noetherian (resp. artinian) modules and the hopfian (resp. co-hopfian) modules.

Finally A. Kaidi introduced in 2009 in [2] the class of endo-noetherian (resp. endo-artinian) modules as follows: an $R$-module $M$ is said to be endonoetherian (resp. endo-artinian) if for every endomorphism $f_{i} \in M$, then $\operatorname{Kerf}_{1} \subset \operatorname{Kerf}_{2} \subset \cdots$ (resp. $\left.I m f_{1} \supset \operatorname{Imf}_{2} \supset \cdots\right)$ is stationary. He showed that the class of endo-noetherian (resp. endo-artinian) modules is between the class of noetherian (resp. artinian) modules and the strongly hopfian (resp. strongly co-hopfian) modules.

The purpose of this paper is to characterize rings on which an endo-noetherian module $M$ is noetherian. Such rings will be called EKFN-rings. We prove that a commutative ring with ascending chain condition on annihilators is an $E K F N$-ring if and only if it is an artinian principal ideal ring.

Our paper is structured as follows: the first part covers the properties and basic aspects of certain classes of modules and rings; special attention is paid to the properties of endo-noetherian modules. In the second part, we characterize commutative $E K F N$-ring satisfying ascending chain condition on annihilators.

\section{Preliminaries}

The rings considered in this section are associative with unit. Unless otherwise mentioned, all the modules considered are left unitary modules. 
Definition 2.1. Given a ring $R$ and an element $r \in R$, let us denote the left translation of $R$ by $\rho_{r}: R \longrightarrow R$. The left annihilator of $r \in R$, denoted by $l(r)=K \operatorname{Ker} \rho_{r}=\{x \in R: x \cdot r=0\}$.

We shall need

Proposition 2.1. ([1], P.203) $\operatorname{End}\left({ }_{R} R\right)=\left\{\rho_{r}: r \in R\right\}$.

Proof. We know that $\left\{\rho_{r}: r \in R\right\} \subset \operatorname{End}\left({ }_{R} R\right)$. Let us show that

$$
\operatorname{End}\left({ }_{R} R\right) \subset\left\{\rho_{r}: r \in R\right\} .
$$

Let $f \in \operatorname{End}\left({ }_{R} R\right), \forall x \in R$, $f(x)=f(x \cdot 1)=x \cdot f(1)$, Let $c=f(1) \in R$, so $x \cdot f(1)=x \cdot c=\rho_{c}(x)$. Then $f=\rho_{c} \in\left\{\rho_{r}: r \in R\right\}$. Thus $\operatorname{End}\left({ }_{R} R\right)=\left\{\rho_{r}: r \in R\right\}$.

Definition 2.2. A module $M$ is called simple if $M \neq 0$ and has no submodules excepted 0 and $M$.

\section{Proposition 2.2. (Schur's Lemma)}

If $S$ and $T$ are two simple modules. Then every non-zero homomorphism is an isomorphism.

Definition 2.3. Let $\left(S_{i}\right)_{i \in I}$ be an indexed set of simple submodules of $M$. If $M$ is the direct sum of this set, then $M=\bigoplus_{i \in I} S_{i}$ is a semisimple decomposition of $M$. A module $M$ is said to be semisimple in case it has a semisimple decomposition.

A ring $R$ is called semisimple if it has a semisimple decomposition.

Definition 2.4. The ring $R$ is said to satisfy the ascending chain condition (acc) on left ideals if every ascending chain of left ideals $I_{1} \lesseqgtr I_{2} \lesseqgtr \cdots \lesseqgtr I_{n} \lesseqgtr \cdots$ becomes stationary.

Definition 2.5. The ring $R$ is said to satisfy the descending chain condition (dcc) on left ideals if every descending chain of left ideals $\cdots \lesseqgtr I_{n} \cdots \lesseqgtr$ $I_{2} \lesseqgtr I_{1}$ becomes stationary.

Definition 2.6. An $R$-module $M$ is called noetherian (resp. artinian) if the set of all submodules of $M$ satisfies the acc (resp. dcc).

By definition, $R$ is an artinian ring if and only if the module ${ }_{R} R$ is artinian.

Theorem 2.3. (see [8], pp. 68).

For a ring $R$ the following statements are equivalent:

1. $R$ is noetherian and every prime ideal is maximal; 
2. $R$ is artinian.

Definition 2.7. Let $N$ and $P$ be $R$-submodules of $M$ such that $M=$ $N \oplus P$. And let $\pi_{N}$ and $\pi_{P}$ denote the respective projection of $M$ on $N$ and $P$.

It is obvious that $\pi_{N}$ and $\pi_{P}$ satisfy:

(i) $\pi_{N}^{2}=\pi_{N} \circ \pi_{N}=\pi_{N}$ and $\pi_{P}^{2}=\pi_{P}$;

(ii) $\pi_{N}+\pi_{P}=I d_{M}$;

(iii) $\pi_{N} \circ \pi_{P}=\pi_{P} \circ \pi_{N}=0$;

(iv) $I m \pi_{N}=\operatorname{Ker} \pi_{P}=N$ and $\operatorname{Ker} \pi_{N}=\operatorname{Im} \pi_{P}=P$.

Definition 2.8. A module $M$ is called hopfian if every surjective endomorphism of $M$ is an automorphism.

Definition 2.9. An $R$-module $M$ is called strongly hopfian if for every endomorphism $f$ of $M$ the ascending chain $\operatorname{Kerf} \subset \operatorname{Ker} f^{2} \subset \cdots \subset \operatorname{Ker} f^{n} \subset$ -.. stabilizes.

If ${ }_{R} R$ is strongly hopfian, then we say that the ring $R$ is left strongly hopfian.

Proposition 2.4. [6] Let $M$ be a module. If $M$ is strongly hopfian then $M$ is hopfian.

Proof. Assume that $M$ is strongly hopfian, and let $f$ be a surjective endomorphism of $M$. Then, there exists $n \geq 1$ such that $K e r f^{n}=K \operatorname{Kerf} f^{n+1}$. Let $x \in M$, such that $f(x)=0$; we know that $f$ is surjective, so is $f^{n}$. And then there exists $y \in M$ such that $x=f^{n}(y)$. Then, $y \in K \operatorname{Kerf^{n+1}}=K \operatorname{Ker} f^{n}$. So $x=0$, thus $f$ is an automorphism.

Definition 2.10. $\quad R$ is called an $S$-ring if every hopfian $R$-module is noetherian.

Proposition 2.5. ([3], P.252) A commutative artinian ring with principal ideal is an $S$-ring.

Definition 2.11. An $R$-module $M$ is called endo-noetherian if any ascending chain of endomorphic kernels $\operatorname{Ker} f_{1} \subset K \operatorname{Ker} f_{2} \subset \cdots \subset K \operatorname{Ker} f_{n} \subset \cdots$ stabilizes, where $f_{i}$ 's are endomorphisms of $M$, ie there exists a positive integer $n$ such that $\operatorname{Ker} f_{n}=\operatorname{Kerf_{n+1}}$.

Proposition 2.6. [2] Every noetherian $R$-module $M$, is endo-noetherian.

Proof. The assertion is a consequence of the fact that $\operatorname{Kerf}$ is a submodule of $M$. 
Proposition 2.7. $[2] \mathbb{Q}$ is a non-noetherian $\mathbb{Z}$-module which is endonoetherian.

Proof. Let $f$ be a non-zero $\mathbb{Z}$-homomorphism of $\mathbb{Q}$. If $x \in \operatorname{Ker} f$, then $f(x)=0$. But $x \in \mathbb{Q}$, so $x=\frac{a}{b}$, where $a \in \mathbb{Z}, b \in \mathbb{Z}^{*}$. We have $f\left(\frac{a}{b}\right)=$ $f\left(a \cdot \frac{1}{b}\right)=a \cdot f\left(\frac{1}{b}\right)=0$. Then $a=0$ or $f\left(\frac{1}{b}\right)=0$. Assume that $f\left(\frac{1}{b}\right)=0$, then $0 \neq f(1)=f\left(\frac{b}{b}\right)=f\left(b \cdot \frac{1}{b}\right)=b \cdot f\left(\frac{1}{b}\right)=0$, contradiction. So $a=0$. Then $x=0$, ie $\operatorname{Kerf}=0$. In fact every non-zero $\mathbb{Z}$-homomorphism of $\mathbb{Q}$ is a monomorphism. So every ascending chain $\operatorname{Kerf}_{1} \subset \operatorname{Kerf} f_{2} \subset \cdots \subset \operatorname{Kerf} f_{n} \subset \cdots$ is stationary, where $f_{i} \in \operatorname{End}(\mathbb{Q})$. Then $\mathbb{Q}$ is endo-noetherian. But $\mathbb{Q}$ is non-noetherian, because of the fact that the sequence $\left(2^{-n} \mathbb{Z}\right)_{n \geq 0}$ is strictly increasing.

Proposition 2.8. [2] A ring $R$ is endo-noetherian if and only if $R$ satisfies the ascending chain condition on principal left annihilators.

Proof. Assume that $R$ is endo-noetherian, let $l\left(a_{1}\right) \subset l\left(a_{2}\right) \subset \cdots \subset l\left(a_{n}\right) \subset$ $\cdots$ be an ascending chain for principal annihilators. We know by definition 2.1 that $l\left(a_{n}\right)=\operatorname{Ker} \rho_{a_{n}}=\left\{x \in R: x \cdot a_{n}=0\right\}$. Then we obtain this new chain $\operatorname{Ker} \rho_{a_{1}} \subset \operatorname{Ker} \rho_{a_{2}} \subset \cdots \subset \operatorname{Ker} \rho_{a_{n}} \subset \cdots$ which stabilizes. Conversely if $R$ satisfies the ascending chain condition on principal annihilators, then by proposition 2.1, for every endomorphism $f_{i}$, there exists $r_{i} \in R$ such that $f_{i}=$ $\rho_{r_{i}}$. And the result follows.

Proposition 2.9. [2] Let $M$ be an endo-noetherian $R$-module. Then $M$ satisfies the ascending chain condition on direct summands.

Proof. Let $N_{1} \subset N_{2} \subset \cdots \subset \cdots$ be an ascending chain of direct summands. $N_{i}$ is a direct summand of $M$, then there exists a submodule $P_{i}$ of $M$ such that: $M=N_{i} \oplus P_{i}$. And then, there exists a map $\pi_{P_{i}}$ such that $\operatorname{Ker} \pi_{P_{i}}=N_{i}$ by 2. Thus we obtain this new chain $\operatorname{Ker} \pi_{P_{1}} \subset \operatorname{Ker} \pi_{P_{2}} \subset \cdots \subset \cdots$ which is stationary.

Corollary 2.1. [2] Let $M$ be an endo-noetherian $R$-module that is a direct sum of non-zero submodules, $M=\bigoplus_{i \in I} M_{i}$. Then the set $I$ is finite.

Proof. Taking into account that $M_{1} \subset M_{1} \oplus M_{2} \subset \cdots \subset \cdots$ is an ascending chain on direct summands. By proposition 2.9, this chain stabilizes.

Remark 2.1. Let $M$ be an $R$-module. If $M$ is endo-noetherian, then $M$ is strongly hopfian.

Proposition 2.10. [2] Let $M$ be an $R$-module and $N$ a submodule of $M$ : if $M$ is endo-noetherian and $N$ is a direct summand then $N$ is endo-noetherian. 
Proof. Assume that $M=N \oplus L$ and let $\operatorname{Kerh}_{1} \subset \operatorname{Kerh}_{2} \subset \cdots \subset K e r h_{n} \cdots$ be an ascending chain, where the $h_{i}$ 's are endomorphisms of $N$ that can be extended to $M$ as $f_{i}=h_{i} \oplus 0,0$ being the zero endomorphism of $L$. Since $M$ is endo-noetherian, there exists $n \in \mathbb{N}$ such that $\operatorname{Ker} f_{n}=K \operatorname{Ker} f_{n+1}$. Then $K e r h_{n}=K e r h_{n+1}$. Thus, $N$ is endo-noetherian.

Proposition 2.11. [2] Let $M_{i}, i \in I$, be a family of $R$-modules such that the set $\operatorname{Hom}\left(M_{i}, M_{j}\right)=\{0\}$ for $i \neq j$ and let $M=\bigoplus_{i \in I} M_{i}$. Then $M$ is endo-noetherian if and only if for every $i \in I$ the module $M_{i}$ is endo-noetherian.

Proof. By proposition 2.10, if $M$ is endo-noetherian, so is $M_{i}$.

Assume now that every $M_{i}$ is endo-noetherian. Since $\operatorname{Hom}\left(M_{i}, M_{j}\right)=$ 0 for all $i \neq j$, we have $\operatorname{End}(M) \cong \prod_{i \in I} \operatorname{End}\left(M_{i}\right)$. And if $f=\left(f_{i}\right)$ is an endomorphism of $M$, then we have that $f\left(\sum a_{i}\right)=\sum f_{i}\left(a_{i}\right)$ : this sum is well defined because of the fact that almost all $a_{i}$ vanish. Then $f=\bigoplus_{i \in I} f_{i}$. For every endomorphism $f \in \operatorname{End}(M)$ we have $\operatorname{Ker} f=\bigoplus_{i \in I} \operatorname{Ker} f_{i}$. Then, every ascending chain of kernels of $\operatorname{End}(M)$ gives a chain of the same type in each $\operatorname{End}\left(M_{i}\right)$, that stabilizes. Thus $M$ is endo-noetherian.

\section{The Main Results}

Let $R$ be an associative and commutative ring with identity $1 \neq 0$.

Definition 3.1. $R$ is said to be an $E K F N$-ring if every endo-noetherian $R$-module is noetherian.

Example 3.1. A semisimple ring is an $E K F N$-ring.

Proposition 3.1. Every homomorphic image of an EKFN-ring is an EKFN-ring.

Proof. Let $R$ be an $E K F N$-ring and $\varphi: R \longrightarrow S$ a surjective homomorphism of rings. Let $M$ be an $S$-module, so $\varphi$ induces a structure of $R$ module on the additive abelian group $M$ by the following map: $R \times M \longrightarrow M$ $(r, m) \mapsto \varphi(r) \cdot m$, and then any $S$-endomorphism is an $R$-endomorphism, and conversely.

Assume that $M$ is an endo-noetherian $S$-module and let's show that $M$ is noetherian. Let $\operatorname{Ker} f_{1} \subset \operatorname{Ker} f_{2} \subset \cdots \subset \operatorname{Ker} f_{n} \subset \cdots$ be an ascending chain for 
$R$-endomorphic kernels, where $f_{i} \in \operatorname{End}_{R}(M)$. Every $R$-endomorphism $f_{i}$, is an $S$-endomorphism, then the ascending chain for $R$-endomorphic is an ascending chain for $S$-endomorphic which stabilizes. So $M$ is an endo-noetherian $R$ module, then $M$ is noetherian.

Proposition 3.2. Any integral domain $E K F N$-ring is a field.

Proof. Let $K=S^{-1} R$ be the field of fractions of $R$, where $S=R-\{0\}$. We know that $K$ has an $R$-module structure. Let $f$ be an $R$-endomorphism, then $\operatorname{Kerf}=0$ or $\operatorname{Kerf}=K$. It follows that any ascending chain for endomorphic kernels of $K$ stabilizes. Then $K$ is noetherian. We know that the map $i$ : $R \longrightarrow K$ is a monomorphism, so $R \subset K$. K is noetherian, then $K$ is a finitely generated $R$-module, so $K$ is a fractional ideal of $R$ by ([11], P.134). And then there exists $r \in R$ such that $r K \subset R$. But $r K=K$, then $K \subset R$.

Proposition 3.3. Any prime ideal of an EKFN-ring is maximal.

Proof. Let $P$ be a prime ideal of $R$ and let $s$ be the canonical surjection defined by $R \longrightarrow R / P r \mapsto \tilde{r}$. By proposition 3.1 the quotient ring of the integral domain $R / P$ is an $E K F N$-ring, then $R / P$ is a field. So $P$ is maximal. $\square$

Proposition 3.4. Let $R$ be an EKFN-ring. Then the set of all maximal ideals of $R$ is finite.

Proof. Let $L$ be the set of all primes ideals of $R$. Let us show that

$$
\operatorname{Hom}_{R}\left(R / P_{i}, R / P_{j}\right)=\{0\} \quad \forall i \neq j .
$$

Let $f \in \operatorname{Hom}_{R}\left(R / P_{i}, R / P_{j}\right)$, defined by $f: R / P_{i} \longrightarrow R / P_{j} a+P_{i}=\tilde{a}_{P_{i}} \mapsto \tilde{a}_{P_{j}}$, where $i \neq j$. It is easy to check that $f$ is an $R$-homomorphism, let's show that $f$ is zero. For every prime ideal $P_{i}, R / P_{i}$ is a simple $R$-module, and by Schur's Lemma $f$ is zero or an isomorphism. Assume that $f$ is an isomorphism. Let $a \in\left(P_{j}-P_{i}\right)$, we have $\tilde{0}_{P_{j}} \neq f\left(\tilde{a}_{P_{i}}\right)=f\left(a \cdot \tilde{1}_{P_{i}}\right)=a \cdot f\left(\tilde{1}_{P_{i}}\right)=a \cdot \tilde{1}_{P_{j}}=\tilde{a}_{P_{j}}=\tilde{0}_{P_{j}}$, contradiction. Then $f=0$, so $\operatorname{Hom}_{R}\left(R / P_{i}, R / P_{j}\right)=\{0\}$. Let $M=\bigoplus_{i \in I} R / P_{i}$, let's show that $M$ is endo-noetherian. We know that $R / P_{i}$ is endo-noetherian. By proposition 2.11, $M$ is endo-noetherian, then the set $I$ is finite.

Proposition 3.5. An artinian ring with principal ideal is an EKFN-ring.

Proof. Let $M$ be an endo-noetherian $R$-module, so $M$ is strongly hopfian, and by proposition $2.4 M$ is hopfian. Thus $M$ is noetherian, by proposition 2.5 .

Proposition 3.6. Let $R$ be a ring with ascending chain condition on principal left annihilators, if $R$ is an EKFN-ring, then $R$ is artinian. 
Proof. By proposition $2.8 R$ is endo-noetherian, then $R$ is noetherian. We know by proposition 3.3 that any prime ideal of an $E K F N$-ring is maximal. Then $R$ is artinian.

Lemma 3.1. ([3], P.248)

Let $R$ be an artinian ring having at least one non-principal ideal. Then $R$ admits a local quotient ring $B$ with maximal ideal $J$ such that $J^{2}=(0)$, and such that $J / J^{2}$ is a $B / J$-vector space of dimension 2.

Lemma 3.2. ([3], P.248)

Let $R$ be an artinian ring having at least one non-principal ideal. Then $R$ admits a quotient ring $B=C \oplus b C$, where $C$ is a local subring of $B$, with maximal ideal $r C \neq 0$, and $b \neq 0$ with $r^{2}=r b=b^{2}=0$.

Lemma 3.3. ([3], P.249)

Let $C$ be a local ring with maximal ideal $r C \neq 0$, where $r^{2}=0$. Let $M$ be the total ring of fractions of the ring of polynomials $C[X]$, and $\sigma$ the $C$-endomorphism of $M$ defined for all $m \in M$, by $\sigma(m)=r X m$, Then:

1. $r \sigma=\sigma^{2}=0$;

2. If $F$ is a $C$-endomorphism of $M$ commuting with $\sigma$, then for all $m \in$ $M, F(r m)=r m F(1)$.

Proposition 3.7. Let $C$ be a local ring with maximal ideal $r C \neq 0$, where $r^{2}=0$. Let $M$ be the total ring of fractions of the ring of polynomials $C[X]$, and $\sigma$ the $C$-endomorphism of $M$ defined for all $m \in M$, by $\sigma(m)=r X m$. Then every non-zero $C$-endomorphism $F$ of $M$ commuting with $\sigma$ is such that either $\operatorname{Ker} F=r M$, or is a monomorphism.

Proof. Note that a ring element $m \in M$ is invertible if and only if $m \notin r M$. If $F(1)$ is not invertible, then $F(1) \in r M$. For any $m \in r M$ there exists $q \in M$ such that $m=r q$. Hence $F(m)=F(r q)=r q F(1)=m F(1)=0$. So $r M \subset \operatorname{KerF}(1)$. Let's show that in this case for all $m \notin r M$, then $F(m) \neq 0$. We have for all $m \in M \backslash r M, r m \in r M \backslash\{0\}$, then $F(r m)=r m F(1)=0$. Or $F(r m)=r F(m)=0$, so $F(m) \in r M$ ie there exists $\alpha \in M$ such that $F(m)=r \alpha$. Assume that $\alpha \in r M$, so $F(m)=0$. Then $m \in K \operatorname{Ker} F$, ie $M \backslash r M \subset \operatorname{KerF}(2)$. Hence (1) and (2) imply that $M \subset \operatorname{KerF}$, then $F$ is identically zero (impossible by assumption). Hence, it follows that $\alpha \notin r M$. So $F(m)=r \alpha \neq 0$. Then, $\forall m \notin r M F(m) \neq 0$. Hence $m \in K e r F \Rightarrow m \in r M$ so $K e r F \subset r M$ (3). So (1) and (3) imply that $\operatorname{KerF}=r M$.

If $F(1)$ is invertible, ie $F(1) \notin r M$. Let $m$ be a non-zero element of $M$. Let's show that $F(m) \neq 0$. In case $m \in r M$, then $F(m)=m F(1)$. Then $F(m) \neq 0$. 
If now $m \notin r M$, then $r m \neq 0$, and then $F(r m)=r m F(1)$, hence $F(r m)=$ $r F(m)=r m F(1) \neq 0$. Then $F(m) \neq 0$. Thus $F$ is a monomorphism.

Proposition 3.8. ([3], P.250) Let $R$ be an artinian ring having at least one non-principal ideal. Then there exists an $R$-module $M$ which is not finitely generated.

Proof. By lemma 3.2, assume that $R=C \oplus b C$, where $C$ is a local subring of $R$, with maximal ideal $r C \neq 0$, and $b \neq 0$ with $r^{2}=r b=b^{2}=0$. Let $M$ be the total ring of fractions of the ring of polynomials $C[X]$ and $\varphi$ the map defined by: $R \longrightarrow \operatorname{End}(M) \alpha+\beta b \mapsto \alpha 1_{M}+\beta \sigma$, where $\alpha, \beta \in C, 1_{M}$ the identity map of $M$ and $\sigma$ the $C$-endomorphism defined in lemma 3.3. $\varphi$ is a ring homomorphism which gives to $M$ an R-module structure and for this structure, $R$-endomorphism of $M$ are $C$-endomorphism of $M$ which commute with $\sigma$. To show that $M$ is not a finitely generated $R$-module, it suffices to note that $M$ contains infinite direct sum of non-zero submodules $\bigoplus_{n \geq 1} R r X^{n}$.

Theorem 3.9. Let $R$ be an artinian ring having at least one non-principal ideal. Then there exists an endo-noetherian $R$-module $M$ which is non-noetherian.

Proof. By proposition 3.8, there exists an $R$-module $M$ which is not finitely generated. Then $M$ is non-noetherian. Let $\operatorname{Kerf}_{1} \subset \operatorname{Kerf_{2}} \subset \cdots \subset \operatorname{Kerf}_{n} \subset$ $\cdots$ be an ascending chain for $R$-endomorphic kernels, where $f_{i} \in \operatorname{End}_{R}(M)$. We know that $R$-endomorphism of $M$ are $C$-endomorphism of $M$ which commute with $\sigma$, then any ascending chain for $R$-endomorphic stabilizes. Thus $M$ is endo-noetherian.

\section{Theorem 3.10. Characterization theorem}

Let $R$ be a ring satisfying acc on annihilators, the following conditions are equivalent:

1. $R$ is an EKFN-ring;

2. $R$ is an artinian principal ideal ring.

Proof.

- Assume that $R$ is an $E K F N$-ring. Since $R$ satisfies the acc on annihilators, then by proposition 3.6, $R$ is artinian. Assume that $R$ has at least one non-principal ideal, so there exists an endo-noetherian module which is non-noetherian by theorem 3.9, contradiction. Thus $R$ is an artinian principal ideal ring. 
- Assume that $R$ is an artinian principal ideal ring. Then by proposition $3.5, R$ is an $E K F N$-ring.

\section{References}

[1] A. Hmaimou, A. Kaidi, E. Sánchez Campos, Generalized Fitting modules and rings, Journal of Algebra, 308 (2007), 199-214.

[2] A. Kaidi, Modules with chain conditions on Endoimages Endokernels, Preprint.

[3] A. Kaidi, M. Sangharé, Une caractérisation des anneaux artiniens à idéaux principaux, Lecture Notes in Math (Springer-Verlag), 1328 (1988), 245254.

[4] C.T. Gueye, M. Sangharé, On commutative FGS-rings, Comm. Algebra, 32, No.5 (2004), 1715-1727.

[5] E. Noether, Idealtheorie in Ringbereichen, Mathematische Annalen, 83 (1921), 24-66.

[6] F.W. Anderson, K.R. Fuller, Rings and Categories of Modules, Grad. Texts in Math vol.13 Springer-Verlag, New York (1992).

[7] G. Baumslag, Hopficity and abelian groups, Topics in abelian groups, Scott Foresman and Company, (1963), 331-335.

[8] J.P. Lafon, Anneaux locaux commutatifs sur lesquels tout module de type fini est somme directe de modules monogènes, J. of Algebra, 17 (1971), 575-591.

[9] M. Barry, O. Diankha, M. Sangharé, On commutative FGI-rings, Math.Sci.Res.J, 9, No. 4 (2005), 87-91.

[10] M. Sangharé, Sur quelques classes d'anneaux liées au lemme de Fitting, Rend.sem.Mat.Univ.Padova, 87 (1992), 29-37.

[11] M.P. Malliavin, Algèbre commutative: Applications en géométrie et théorie des nombres, Masson, (1985). 
